\title{
Effect of Personality Trait and Risk Level on Generation and Intensity of Regret
}

\author{
Yaozhong Liu, Xiyong Cao, Min Liu \\ Management School, Jinan University, Guangzhou, China \\ Email: 282712903@qq.com
}

Received 18 November 2014; revised 19 December 2014; accepted 27 December 2014

Copyright (C) 2015 by authors and Scientific Research Publishing Inc.

This work is licensed under the Creative Commons Attribution International License (CC BY). http://creativecommons.org/licenses/by/4.0/

(c) (i) Open Access

\begin{abstract}
Our research explored the effect of personality trait and risk level to generation and intensity of regret. The results showed: 1 ) The main effect of the level of risk was significant. At high risk level, subjects generated higher frequency and intensity of regret than at low risk level. However, the main effect of personality trait was not significant; 2 ) Interaction of personality trait and risk level was significant. At high risk level, pessimistic subject generated higher intensity regret than at low risk level while the intensity of regret of optimistic subject did not show a significant difference at different risk levels.
\end{abstract}

\section{Keywords}

Regret, Personality Trait, Risk Level, Risk Decision

\section{Introduction}

Regret is a negative emotion which individual often experiences in daily life. Each of us will encounter such a scenario: After we make a decision, no matter how reasonable this decision seemed at that time, we will generate the "if-only" idea when we look back and the emotion with this idea is regret. (Ming Wu, 2013). Kahneman and Tversky (1982) researched the influencing factors of regret and put forward the action theory that faced with the same decision-loss result, the intensity of regret resulting from "action" is significantly higher than resulting from "inaction" [1]. They put forward "Normal Theory" based on the "action effect", which emphasizes that the criteria of events can be in before or after the event and there is a process of comparison. Generally, "inaction" is a normal choice while "action" is beyond the routine, so that people would feel greater intensity of regret if they did something. Also, the theory suggests that because the events are often evaluated through comparing it with different standards in different backgrounds, the results of evaluation are varied. When the results of the action are easy to be imaged, the response to the results is strengthened and frequency and intensity resulting 
from "action" is higher than "inaction". Landman and Zeelerberg (1999) attributed this phenomenon to the process of attribution. Because the result resulting from "action" is more attributed to self-responsibility, it causes more intense emotion [2]. Recently, the main trend in regret research has focused on the study of personality trait, situational information and neural mechanism. Putten, Zeelenberg and van Dijk (2009) found that personality orientation affects the generation of regret. People with state orientation are easier immersed into regret mood than people with behavior orientation. Kuhnle and Sinclair (2011) found that decision-making style influenced the generation and intensity of regret [3]. Chinese scholars' research shows that attribution style can also influence the regret after decision. Personality provides a basis to the generation of regret and contextual information plays a promoting and facilitating role in the generation of regret (Hong Mei Gao, Yan Zhang, 2013).

Synthesizing the domestic and foreign research, influencing factors of regret include individual factors, such as personality trait, achievement motivation, risk appetite, attribution, etc., and property of decision-making information, such as the degree of importance, the level of risk, the number of information, etc. However, previous researches are less mentioned the effect of optimistic personality and risk level to regret which are very common in daily life [4]. Yong Zhu and Zhan Xu (2011) indicated that high-risk decision was with high yield but at the same time with high loss. Therefore, high loss will bring large psychological impact which causes negative emotion such as regret. While low-risk decision is not easy to produce negative emotions [5], Carr's research (2011) showed that optimistic people attributed negative events, negative experiences and setbacks to external, temporary and specific factors which did not have significance of widespread value. Just because they attribute failure to external factors, the negative feeling can dissipate quickly [6].

In summary, we predict that in decision-making situations, pessimistic decision maker makes pessimistic attribution which results in higher regret while optimistic decision maker makes optimistic attribution which results in lower regret. At the same time, because of the difference of attribution, pessimistic decision maker experiences larger psychological impact than optimistic decision maker in the case of larger loss. Therefore, our research investigates the relationship of optimistic personality and generation and intensity of regret. Further, we investigate whether personality trait adjusts the effect of risk level to generation and intensity of regret. Therefore, this research presents the following hypothesis:

Hypothesis 1: The main effect of personality trait is not significant. Optimistic/pessimistic personality affects the generation and intensity of regret;

Hypothesis 2: The main effect of the risk level is significant. Risk level affects generation and intensity of regret;

Hypothesis 3: Interaction between personality trait and risk level is significant. Pessimistic people experience higher regret at high risk decision situation than optimistic people.

\section{Method}

\subsection{Participants}

Thirty-four postgraduates from JiNan University (19 females and 15 males; mean age 23.92 years, SD = 2.00) participated in the experiment. Optimism Personality Scale was used to distinguish optimistic/pessimistic personality. 160 valid questionnaires were issued, using $27 \%$ Standard to distinguish optimistic/pessimistic group.

\subsection{Research Design}

The experiment had a $2 \times 2$ two factor mixed experimental design, with the first factor referring to personality trait (optimistic personality vs. pessimistic personality) and the second factor referring to risk level (high risk level vs. low risk level). The first factor was between-participant design and the second factor was within-participant design factor. The dependent variable was the frequency and intensity of regret through participant's subjective assessment.

\subsection{Research Tools}

Optimism Personality Scale (OPS). OPS is compiled by Yilong Li through adding homogeneous questions into Life Orientation Test Revision (LOT-R). There are 12 items in the OPS. The 1st, 2nd, 3rd, 4th, 6th, 9th, 12th questions used reverse scoring. Total score was arranged from low to high and the former $27 \%$ was treated as optimistic group while latter $27 \%$ were treated as pessimistic group. The internal consistency reliability is 0.799 
which reaches the requirement of surveying. The correlation coefficient of OPS and LOT-R is 0.654, indicating that OPS has good validity.

\subsection{Research Procedure}

Experiments were divided into two phases. First, we selected qualified participant through OPS and then participants conducted gambling experiment in real situation.

Select participant. We dispensed 160 OPSs and recycled 153 valid questionnaires. The 1st, 2nd, 3rd, 4th, 6th, 9th, 12th questions used reverse scoring. Total score was arranged from low to high and the former $27 \%$ was treated as optimistic group $(\mathrm{N} 1=41, \mathrm{M} 1=22.37, \mathrm{SD} 1=0.476)$ while latter $27 \%$ were treated as pessimistic group $(\mathrm{N} 2=41, \mathrm{M} 2=46.39, \mathrm{SD} 2=0.785)$. Independent sample t-test $(\mathrm{t}=-26.18, \mathrm{p}<0.01)$ revealed that the difference of two group was significant. Finally, there were 34 people participated experiment voluntarily. Optimistic group had 17 participants (8 males, 9 females, $22.59 \pm 0.88$ total score) and pessimistic group had 17 participants (7 males, 10 females, $46.29 \pm 1$.23). Independent sample $t$-test $(t=-15.71, p<0.01)$ revealed that the difference of two group was significant.

Gambling experiment. Experiment was operated by computer and experimental program was prepared by Eprime 2.0. After the experiment began, two identical cards showed in screen, left one representing high risk scheme (gain 500 yuan or lose 500 yuan) and right one representing low risk scheme (gain 100 yuan or lose 100 yuan). Participants were asked to select one and then the results of two schemes were presented simultaneously. After that, participants were asked to choose an emotion word (regret or not regret) to express the feeling of result and assess the intensity of this feeling (1 represents "slightly", 9 represents "very"). Before experiment, we informed participants that the more he/she gained, the better the prize was.

There were four possible outcomes: A $(+500,+100)$, B $(+500,-100)$, C $(-500,+100), \mathrm{D}(-500,-100)$ and each outcome appeared 20 times. So there were 80 trails and when completed 40 trails, participants rested 1 minute. If participants chose high risk scheme, the outcome was $\mathrm{C}$ or $\mathrm{D}$ and if participants chose low risk scheme, the outcome was A or B. So he/she was in the state of loss and might experience regret. Before formal experiment, there were 5 practice trails.

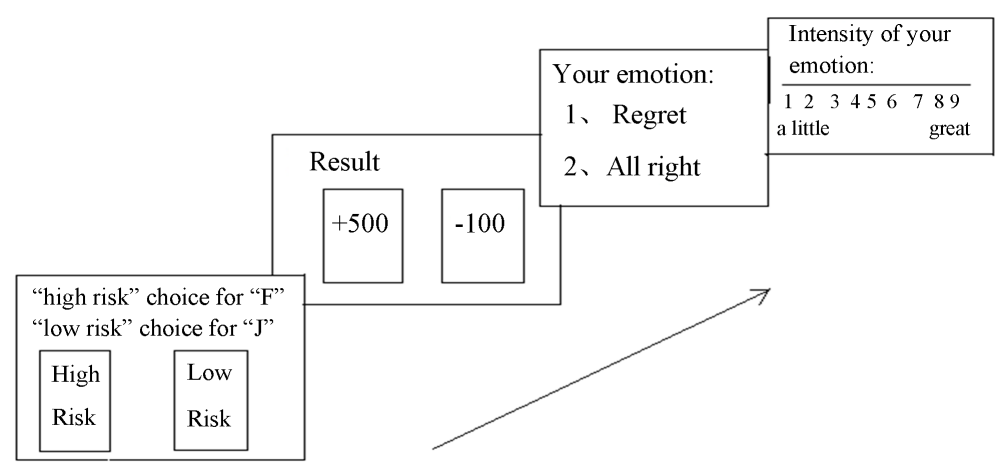

\section{Results}

\subsection{Effect of Personality Trait and Risk Level to Generation of Regret}

SPSS13.0 was used to do homogeneity of test variance for the data in Table 1. The SD. of optimistic group and pessimistic group was homogeneous $(p<0.05)$, so analysis of variance could be used. Repeated measures ANOVA of mixed design was used to test whether personality trait and risk level can affect generation of regret. ANOVA revealed only a significant main effect of risk level $(\mathrm{F}(1,32)=48.50, \mathrm{p}<0.01, \eta 2=0.60)$, indicating that frequency of generation of regret in high risk level $(0.85 \pm 0.25)$ was significantly higher than in low risk level $(0.58 \pm 0.23)$ while main effect of personality trait was not significant $(\mathrm{F}(1,32)=0.46, \mathrm{p}>0.05, \eta 2=0.01)$ and interaction between personality trait and risk level $(\mathrm{F}(1,32)=0.42, \mathrm{p}>0.05, \eta 2=0.01)$ was not significant.

\subsection{Effect of Personality Trait and Risk Level to Intensity of Regret}

SPSS13.0 was used to do homogeneity of test variance for the data in Table 2. The SD. of optimistic group and 
Table 1. Average and standard deviation of effect of different personality trait and risk level to generation of regret.

\begin{tabular}{ccccc}
\hline Risk level & \multicolumn{2}{c}{ Optimistic group } & \multicolumn{2}{c}{ Pessimistic group } \\
\hline & AVE & SD. & AVE & SD. \\
High risk level & 0.84 & 0.26 & 0.86 & 0.25 \\
Low risk level & 0.54 & 0.23 & 0.62 & 0.24 \\
\hline
\end{tabular}

Table 2. Average and standard deviation of effect of different personality trait and risk level to intensity of regret.

\begin{tabular}{ccccc}
\hline Risk level & \multicolumn{2}{c}{ Optimistic group } & \multicolumn{2}{c}{ Pessimistic group } \\
\hline & AVE & SD. & AVE & SD. \\
High risk level & 5.23 & 1.91 & 6.39 & 1.38 \\
Low risk level & 4.86 & 2.61 & 4.87 & 1.59 \\
\hline
\end{tabular}

pessimistic group was homogeneous ( $\mathrm{p}<0.05$ ), analysis of variance could be used. Repeated measures ANOVA of mixed design was used to test whether personality trait and risk level can affect intensity of regret. ANOVA revealed a significant main effect of risk level $(\mathrm{F}(1,31)=13.78, \mathrm{p}<0.05, \eta 2=0.31)$, indicating that intensity of generation of regret in high risk level (5.81 \pm 1.65$)$ was significantly higher than in low risk level $(4.86 \pm 2.10)$ and a significant interaction of personality trait and risk level $(F(1,31)=5.55, p<0.05, \eta 2=0.14)$, as shown in Figure 1. Simple effect test revealed that risk level is significant in pessimistic group $(\mathrm{F}(1,31)=18.53, \mathrm{p}<$ $0.01, \eta 2=0.37$ ), indicating that intensity of regret of pessimistic group in high risk level was higher than in low risk level. Different intensity of regret of different personality in high risk level was marginally significant (F (1, $31)=4.10, \mathrm{p}=0.051, \eta 2=0.12)$. The regret of optimistic group $(5.04 \pm 2.26)$ was lower than pessimistic group (5.63 \pm 1.65$)$.

\section{Discussion}

Our study revealed that risk level significantly affects generation and intensity of regret, indicating that frequency and intensity of regret in high risk level is higher than in low risk level. This shows that the level of risk level in risk decision can serve as an independent risk factor to influence the regret after decision-making defeat. According to Kahneman's Normal theory, the easier the outcome is changed in hypothetical thinking, the stronger emotional reaction is. In this study, if participants choose a low risk decision, he/she will gain a common prize and this can be recognized as a standard. When participants choose a high risk decision, standard has high variability, and then the outcome in hypothetical thinking has high variability, so participants generate counterfactual thinking resulting in higher frequency and intensity of regret [7].

Personality trait can't serve as an independent factor to influence the regret after decision-making defeat but can adjust the effect of risk level to it. According to Standard theory, events are always compared with a standard evaluation in a certain context that different background and standard will result in different outcome. The standard emphasized in this theory can be transcendental or constructed after event. Generation of regret is based on standard constructed after event. Because of the significant difference of construction after event between optimistic group and pessimistic group, there is significant difference of intensity of regret between the two groups in the high risk level. Value of losing 500 yuan is higher in the pessimistic group's eyes than in the optimistic group's eyes, so pessimistic group generates stronger intensity of regret. While because difference of the value of losing 100 yuan is not significant, there is no significant difference of intensity between two groups. So as to frequency of regret, as long as participants lose money in gambling experiment, no matter it is 100 yuan or 500 yuan, regret will be generated.

Our study explored effect of personality trait and risk level to regret after decision-making defeat in the condition of controlling sex and age of participants, obtaining some meaningful finding. But there are some shortcoming in our study to be discussed in the future research. First, our experiment conducted under the real scenarios of gambling task, but we didn't use real money. This may affect the result of the experiment. Second, we limited $+500 /-500$ as high risk level and $+100 /-100$ as low risk level. But the difference between high and low 


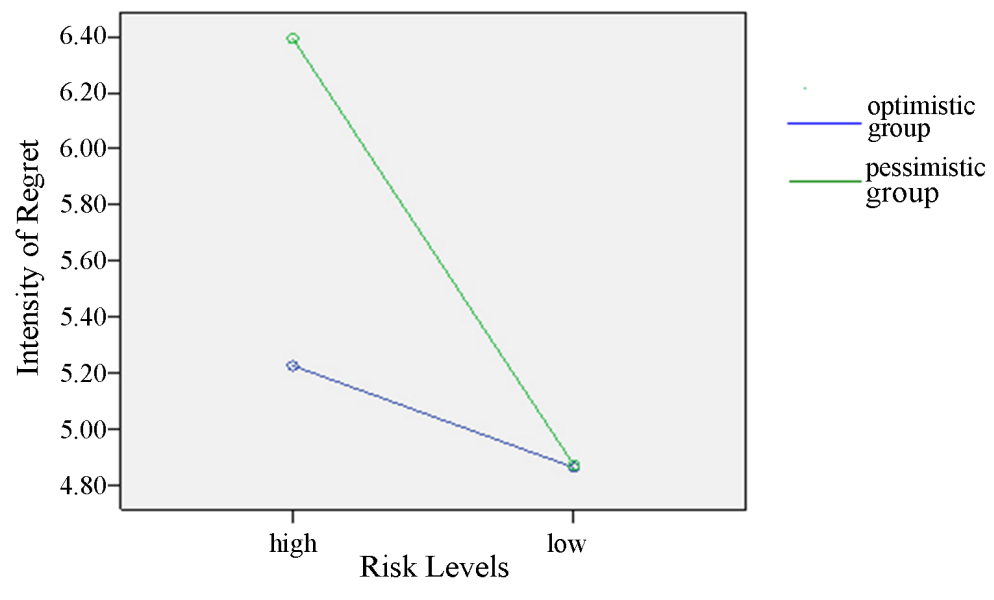

Figure 1. The interaction effects of personality traits and risk levels to Intensity of Regret.

risk level and absolute value of high and low risk level may have different effects to regret. Third, age, sex and education may be the factors to affect the regret after decision-making defeat as well.

\section{References}

[1] Kahneman, D. and Tversky, A. (1982) The Psychology of Preferences. Scientific American, 246, 160-173. http://dx.doi.org/10.1038/scientificamerican0182-160

[2] Connolly, T. and Zeelenberg, M. (2002) Regret in Decision Making. Current Directions in Psychological Science, 11, 212-216. http://dx.doi.org/10.1111/1467-8721.00203

[3] Kuhnle, C. and Sinclair, M. (2011) Decision Mode as an Antecedent of Flow, Motivational Interference, and Regret. Learning and Individual Differences, 21, 239-243. http://dx.doi.org/10.1016/j.lindif.2010.11.024

[4] Gilovich, T. and Medvec, V.H. (1994) The Temporal Pattern to the Experience of Regret. Journal of Personality and Social Psychology, 67, 357-365. http://dx.doi.org/10.1037/0022-3514.67.3.357

[5] Erskine, H. (1973) The Polls: Hopes, Fears, and Regrets. Public Opinion Quarterly, 132-145. http://dx.doi.org/10.1086/268068

[6] Carr, A. (2011) Positive Psychology: The Science of Happiness and Human Strengths. Routledge, London.

[7] Kahneman, D. and Miller, D.T. (1986) Norm Theory: Comparing Reality to Its Alternatives. Psychological Review, 93, 136-153. http://dx.doi.org/10.1037/0033-295X.93.2.136 
Scientific Research Publishing (SCIRP) is one of the largest Open Access journal publishers. It is currently publishing more than 200 open access, online, peer-reviewed journals covering a wide range of academic disciplines. SCIRP serves the worldwide academic communities and contributes to the progress and application of science with its publication.

Other selected journals from SCIRP are listed as below. Submit your manuscript to us via either submit@scirp.org or Online Submission Portal.
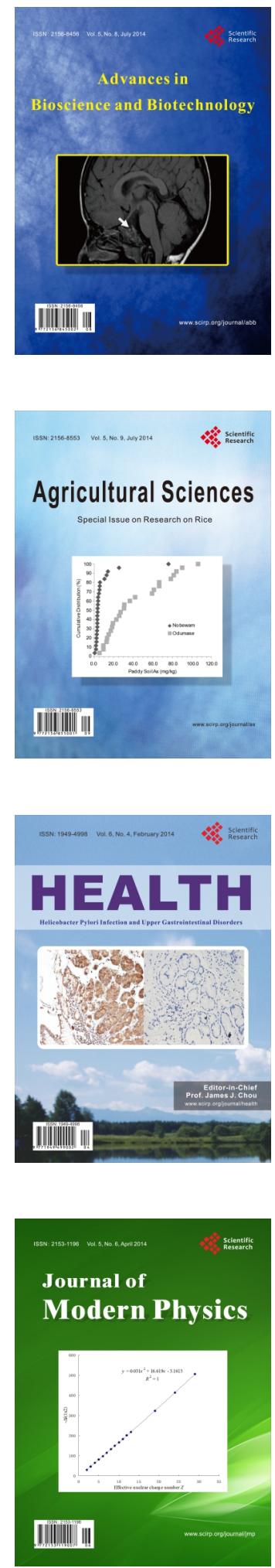
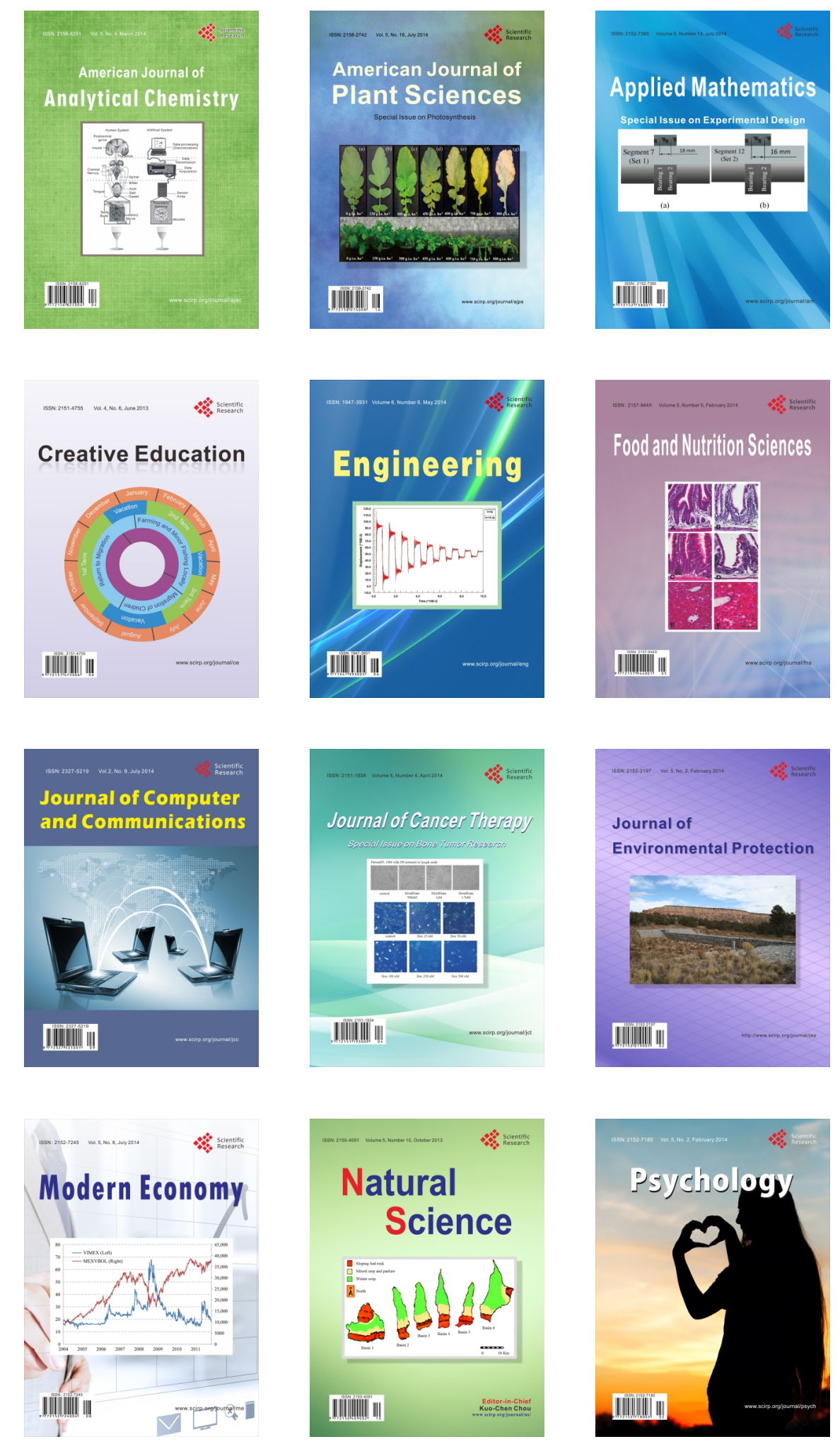